\title{
Adaptive low Mach number simulations of nuclear flame microphysics
}

\author{
J. B. Bell, M. S. Day, C. A. Rendleman \\ Center for Computational Sciences and Engineering \\ Lawrence Berkeley National Laboratory \\ Berkeley, CA 94720 ${ }^{1}$
}

S. E. Woosley and M. A. Zingale

Department of Astronomy and Astrophysics

University of California at Santa Cruz

Santa Cruz, CA, $95064^{2}$

\begin{abstract}
We introduce a numerical model for the simulation of nuclear flames in Type Ia supernovae. This model is based on a low Mach number formulation that analytically removes acoustic wave propagation while retaining the compressibility effects resulting from nuclear burning. The formulation presented here generalizes low Mach number models used in combustion that are based on an ideal gas approximation to the arbitrary equations of state such as those describing the degenerate matter found in stellar material. The low Mach number formulation permits time steps that are controlled by the advective time scales resulting in a substantial improvement in computational efficiency compared to a compressible formulation. We briefly discuss the basic discretization methodology for the low Mach number equations and their implementation in an adaptive projection framework. We present validation computations in which the computational results from the low Mach number model are compared to a compressible code and present an application of the methodology to the Landau-Darrieus instability of a carbon flame.
\end{abstract}

\footnotetext{
1 The work of Bell, Day and Rendleman was supported by the Applied Mathematics Program of the DOE Office of Mathematics, Information, and Computational Sciences under the U.S. Department of Energy under contract No. DE-AC0376SF00098.

2 The work of Woosley and Zingale were supported by the Scientific Discovery through Advanced Computing (SciDAC) program of the DOE, grant number DEFC02-01ER41176 to the Supernova Science Center/UCSC.
} 


\section{Introduction}

Currently, the accepted model for Type Ia supernovae is the explosion of a carbon-oxygen white dwarf. Observational evidence is inconsistent with the nuclear burning occurring in a prompt detonation mode. Detailed computations show that a detonation predicts excess amounts of iron and fails to account for significant amounts of intermediate mass elements observed in the spectra of supernovae events. For this reason, it is believed that at least the initial phases are governed by the propagation of constant-pressure deflagrations. However, to obtain the energy generation rate needed to explode the star the deflagration must be dramatically accelerated relative to the laminar flame speed of the burning front. The recent review article by Hillebrandt and Niemeyer [1] provides an excellent discussion of the issues.

Within the star there are numerous mechanisms that have the potential to accelerate a deflagration wave. Landau-Darrieus $[2,3]$ instabilities can lead to wrinkling of the flame [4]. Because the lighter ash lies below the heavier carbonoxygen fuel, the flame interface is also subject to Rayleigh-Taylor and KelvinHelmholtz instabilities. Finally, the flame can be accelerated by interaction with turbulence arising from convective instabilities within the flame as well as turbulence generated by the deflagration itself.

Efforts focused on understanding the role of the different types of instabilities on accelerating a nuclear flame have generated substantial interest in computational studies of flame microphysics. Several authors have performed simulations in both two and three dimensions based on representing the flame as an interface propagating through the media, see Hillebrandt and Niemeyer [1] for a discussion of this literature. It has also become possible to perform detailed numerical simulations in 2D and 3D that fully resolve the relevant burning and diffusive length scales. Niemeyer and Hillebrandt [5] performed studies of this type but indicate that at the resolutions they present, the effects of numerical diffusion are still apparent. In an effort to model larger physical domains, several investigators have performed resolved computations using modified flame physics. For example, Khokhlov [6] uses an auxiliary variable to model the burning front that predicts the correct 1-d laminar flame speed while thickening the flame. Niemeyer and Hillebrandt [7] and Niemeyer et al. [8] use a weaker nonlinearity in the reaction term to thicken the flame.

Although these types of simulation have been able to provide substantial insight into the dynamics of nuclear deflagrations, they are limited in terms of both the spatial extent that can be modeled and the computational expense associated with long time integrations. The use of modern adaptive mesh methodologies such as FLASH $[9,10]$ can be used to extend the size of the system that can be modeled; however, temporal integration remains a prob- 
lem. The issue arises because the flame phenomena being studied propagate at speeds less than $1 \%$ of the sound speed in the star. Thus, time step limitations based on acoustic Courant-Friedrich-Levy (CFL) considerations severely limit the time step relative to the velocity of the flame.

Our goal in this paper is to introduce a low Mach number formulation of nuclear flames that alleviates the acoustic time step constraint. This approach, based on low Mach number asymptotics, uses a projection formulation coupled with higher-order Godunov advective differencing that allows time-steps based on advection speeds rather than acoustic speeds. This type of approach was first used for combustion by Rehm and Baum [11] and was derived from low Mach number asymptotics by Majda and Sethian [12]. For problems in combustion, governed by an ideal gas equation of state, the low Mach number approach has seen substantial development and has been successfully applied to simulation of laminar flames in one and two dimensions and to threedimensioanl turbulent flames. A complete survey of the combustion literature is beyond the scope of this paper. The reader is referred to Knio et al. [13] and Day and Bell [14] and the references cited in those works for methodology for time-dependent, premixed flames. For steady diffusion flames, see, for example, Bennett and Smooke [15] and Becker et al. [16] and the references cited in these works.

The methodology presented here generalizes the approach of Day and Bell [14] to the nuclear deflagration regime. In particular, we discuss the extension of the low Mach number methodology to degenerate equations of state typical of stellar environments. For applications of this approach see Bell et al. [17] and Bell et al. [18]. We note that in this paper we will focus on fully resolving both the reaction and diffusion length scales; consequently, computational requirements will limit the domains of interest to at best a few meters in each linear dimension. For this reason, we do not need to incorporate the thermodynamics effect of stratification in the star, as represented by an anelastic approximation, in our computations. More precisely, for the domains we consider, the thermodynamic pressure varies by at most 1 part in $10^{6}$ over the domain, so assuming that this pressure is constant for the simulation is a neglible effect. For larger scale computations where the spatial scale ranges over several kilometers, a generalized low Mach number model would be required to accurately capture variations in thermodynamic pressure with altitude.

In the next section we discuss the basic equations and introduce the low Mach number model. In section 3 we discuss the basic projection algorithm and sketch its incorporation into an adaptive mesh refinement algorithm. Section 4 presents a validation of the methodology by comparison with detailed compressible computations and presents an initial application of the method to the study of a Landau-Darrieus instability in two dimensions. In the final section we discuss potential application of this approach to more detailed study 
of nuclear flame acceleration mechanisms.

\section{Low Mach number model}

The low Mach number model is derived from the compressible flow equations using asymptotic analysis. These equations describe conservation of mass, momentum and energy augmented with species equations for the isotopes present in the flame. For the stellar conditions typical of $\mathrm{C}+\mathrm{O}$ flames we are considering here, the Lewis number, which is the ratio of energy transport to species diffusion, is $O\left(10^{7}\right)$ and the Prandtl number, which is the ratio of fluid viscosity to energy transport, is $O\left(10^{-5}\right)$. Under these conditions, the flow is well approximated by the system (see, for example, Timmes and Woosley [19])

$$
\begin{aligned}
\frac{\partial \rho}{\partial t}+\nabla \cdot \rho U & =0 \\
\frac{\partial \rho U}{\partial t}+\nabla \cdot(\rho U U+p) & =\rho \vec{g} \\
\frac{\partial \rho E}{\partial t}+\nabla \cdot(\rho U E+p U) & =\nabla \cdot(\kappa \nabla T)+\rho U \cdot \vec{g}-\sum \rho q_{k} \dot{\omega}_{k} \\
\frac{\partial \rho X_{k}}{\partial t}+\nabla \cdot \rho U X_{k} & =\rho \dot{\omega}_{k}
\end{aligned}
$$

Here, $\rho, U, T$ and $p$ are the density, velocity, temperature, and pressure, respectively, and $E=e+U \cdot U / 2$ is the total energy with $e$ representing the internal energy. In addition, $X_{k}$ is the abundance of the $k^{\text {th }}$ isotope, with associated production rate $\dot{\omega}_{k}$ and energy release $q_{k}$. Finally, $\vec{g}$ is the gravitational force and $\kappa$ is the thermal conductivity. (We note that the assumptions that fluid viscosity and species diffusion are zero can be easily relaxed, see Day and Bell [14]).

For the stellar conditions being considered here the pressure contains contributions from ions, radiation, and electrons. (See Kippenhahn and Weigert [20] for a discussion of equations of state for stellar matter.) Thus,

$$
p=p_{\text {ion }}+p_{\text {rad }}+p_{\text {ele }}
$$

with

$$
p_{\text {ion }}=\frac{\rho k T}{\bar{A} m_{p}} \quad, \quad p_{\text {rad }}=a T^{4} / 3
$$

and $p_{\text {ele }}$ is the contribution to the thermodynamic pressure due to fermions. In these expressions, $m_{p}$ is the mass of the proton, $a$ is related to the Stefan- 
Boltzmann constant $\sigma=a c / 4, c$ is the speed of light, $1 / \bar{A}=\sum_{k} X_{k} / A_{k}$, $A_{k}$ is the atomic number of $k^{\text {th }}$ isotope, and $k$ is Boltzmann's constant. We note that pressure is of the form $p=p\left(\rho, T, X_{k}\right)$. The ionic component has the form associated with an ideal gas but the radiation and electron pressure components do not.

As a prelude to developing the low Mach number equations, we first rewrite the energy equation in terms of the enthalpy, $h=e+p / \rho$

$$
\rho \frac{D h}{D t}-\frac{D p}{D t}=\nabla \cdot \kappa \nabla T-\sum_{k} \rho q_{k} \dot{\omega}_{k}
$$

For the low Mach number asymptotic analysis, we introduce scaled coordinates in which the time scale is proportional to the spatial scale times the advective velocity scale. In this scaling, we expand pressure and velocity in Mach number, $M=U / c_{s},\left(c_{s}\right.$ is the sound speed),

$$
p(x, t)=p_{0}(t)+M p_{1}(t)+M^{2} \pi(x, t)
$$

with a similar equation for $U(x, t)$. Substituting these expansions into the equations of motion given above and matching terms in $M$, we find $p_{1}(t)=0$, and a modified momentum equation:

$$
\frac{\partial \rho U}{\partial t}+\nabla \cdot \rho U U=-\nabla \pi+\rho \vec{g}
$$

Thus, the pressure is decomposed into a thermodynamic component, $p_{0}$, that depends only on time and a perturbation component, $\pi$, that is $O\left(M^{2}\right)$. For the low Mach number model, we ignore the $O\left(M^{2}\right)$ effects on the thermodynamics. For simplicity, in this paper we will assume that the nuclear flame occurs in an open environment under constant pressure so that the thermodynamic pressure is, in fact, a constant which we denote as $p_{0}$. With this assumption, the enthalpy equation reduces to

$$
\frac{\partial \rho h}{\partial t}+\nabla \cdot(\rho U h)=\nabla \cdot \kappa \nabla T-\sum_{k} \rho q_{k} \dot{\omega}_{k}
$$

The enthalpy and momentum equations combined with the species equations (and conservation of mass) describe the evolution of the low Mach number system. However, this evolution is also constrained by the equation of state. We will now show that this constraint is equivalent to a constraint on the divergence of the velocity field. If we differentiate the equation of state along 
particle paths we obtain

$$
0 \equiv \frac{D p}{D t}=\frac{\partial p}{\partial \rho} \frac{D \rho}{D t}+\frac{\partial p}{\partial T} \frac{D T}{D t}+\sum_{k} \frac{\partial p}{\partial X_{k}} \frac{D X_{k}}{D t}
$$

Combining this equation with the mass conservation equation, we obtain

$$
\nabla \cdot U=\frac{1}{\rho \frac{\partial p}{\partial \rho}}\left(\frac{\partial p}{\partial T} \frac{D T}{D t}+\sum_{k} \frac{\partial p}{\partial X_{k}} \frac{D X_{k}}{D t}\right)
$$

To complete the specification of the low Mach number model, we need to derive the temperature evolution equation. We note that although the thermodynamic variables are most naturally expressed here in terms of $\rho, T$, and $X_{k}$, for this derivation, it is more convenient to express the thermodynamics in terms of $p, T$ and the $X_{k}$. With this dependence, differentiating the enthalpy equation we have

$$
\frac{D h}{D t}=\left.\frac{\partial h}{\partial T}\right|_{p, X_{k}} \frac{D T}{D t}+\left.\frac{\partial h}{\partial p}\right|_{T, X_{k}} \frac{D p}{D t}+\left.\sum_{k} \frac{\partial h}{\partial X_{k}}\right|_{p, T, X_{j, j \neq k}} \frac{D X_{k}}{D t}
$$

After substituting from the above equations and using the low Mach number condition on $p$ we have

$$
\rho c_{p} \frac{D T}{D t}=\nabla \cdot \kappa \nabla T-\sum_{k} \rho\left(q_{k}+\xi_{k}\right) \dot{\omega}_{k}
$$

where $\xi_{k}=\left.\frac{\partial h}{\partial X_{k}}\right|_{p, T, X_{j, j \neq k}}$, and $c_{p}=\left.\frac{\partial h}{\partial T}\right|_{p, X_{k}}$ is the specific heat at constant pressure.

Substituting this into the above equation for $\nabla \cdot U$ yields an expression for a constraint on the advective flow velocities:

$$
\nabla \cdot U=\frac{1}{\rho \frac{\partial p}{\partial \rho}}\left(\frac{1}{\rho c_{p}} \frac{\partial p}{\partial T}\left(\nabla \cdot \kappa \nabla T-\sum_{k} \rho\left(q_{k}+\xi_{k}\right) \dot{\omega}_{k}\right)+\sum_{k} \frac{\partial p}{\partial X_{k}} \dot{\omega}_{k}\right) \equiv S .(5)
$$

\section{$3 \quad$ Numerical methodology}

In this section we discuss the numerical methodology used to integrate the low Mach number equations described above. The spatial discretization uses a second-order finite volume Godunov procedure. The temporal discretization strategy is a fractional step approach based on a projection approximation. In 
this approach we integrate the equations for momentum, isotope abundances and enthalpy using a lagged approximation to the constraint. We then apply a discrete projection to the intermediate velocity computed in the first step to enforce the constraint. This basic fractional step algorithm is embedded in a hierarchical adaptive mesh refinement (AMR) algorithm. The version of the methodology presented here is an adaptation of the method presented by Day and Bell [14] for chemical combustion. In the next subsection we describe the single-grid algorithm. We then discuss incorporation of that algorithm into an adaptive projection framework.

Before describing the algorithm, we note that our approach differs from the standard approach to discretizing the low Mach number system originally proposed for combustion by McMurtry et al. [21]. In the McMurtry et al. approach an auxiliary equation for the density in convective form is derived by differentiating the equation of state in time and replacing temporal derivatives of temperature and species by spatial derivatives of these quantities. This equation is then used to advance the density in time with temperature being determined from the equation of state. In the projection step, the McMurtry et al. algorithm solves a constant coefficient Poisson equation to modify the velocity field so that the conservation of mass equation is satisfied. In constrast to this approach, we directly solve the conservation form of the equations for both enthalpy and density. Our projection step solves a variable coefficient elliptic equation to enforce the velocity constraint given in equations 5. Although a comprehensive comparison of these approaches is not available, our approach, although somewhat more expensive, conserves both mass and energy and appears to provide a more robust discretization. See Day and Bell [14] and Nicoud [22] for a more complete discussion of these issues.

\subsection{Single grid algorithm}

The single grid algorithm is essentially a three-step process. First, we use an unsplit second-order Godunov procedure to predict a time-centered $\left(t^{n+1 / 2}\right)$ advection velocity, $U^{\mathrm{ADV}, *}$, using the cell-centered data at $t^{n}$ and the lagged pressure gradient from the interval centered at $t^{n-1 / 2}$. The provisional field, $U^{\mathrm{ADV} \text {,* }}$, represents a normal velocity on cell edges analogous to a MAC-type staggered grid discretization of the Navier-Stokes equations (see Harlow and Welch [23], for example). However, $U^{\mathrm{ADV}, *}$ fails to satisfy the time-centered divergence constraint. We apply a discrete projection by solving the elliptic equation

$$
D^{\mathrm{MAC}} \frac{1}{\rho^{n}} G^{\mathrm{MAC}} \phi^{\mathrm{MAC}}=D^{\mathrm{MAC}} U^{\mathrm{ADV}, *}-\left(S^{n}+\frac{\Delta t^{n}}{2} \frac{S^{n}-S^{n-1}}{\Delta t^{n-1}}\right)
$$


for $\phi^{\mathrm{MAC}}$, where $D^{\mathrm{MAC}}$ represents a centered approximation to a cell-based divergence from edge-based velocities, and $G^{\mathrm{MAC}}$ represents a centered approximation to edge-based gradients from cell-centered data. The solution, $\phi^{\mathrm{MAC}}$, is then used to define

$$
U^{\mathrm{ADV}}=U^{\mathrm{ADV}, *}-\frac{1}{\rho^{n}} G^{\mathrm{MAC}} \phi^{\mathrm{MAC}} .
$$

$U^{\mathrm{ADV}}$ is a second-order accurate, staggered-grid vector field at $t^{n+1 / 2}$ that discretely satisfies the constraint (5), and is used for computing the time-explicit advective derivatives for $U$, $\rho h$ and $\rho X_{k}$.

In the next step of the algorithm we advance the advection-reaction-diffusion system for $\rho h$ and $\rho X_{k}$. For the supernovae flames considered here, the nuclear burning occurs on a scale faster than the fluid dynamics. For that reason, we treat the reactions using a symmetric Strang-splitting approach so that the reactions can be treated with stiff ODE technology. We first advance the reactions terms $\Delta t / 2$ in time. We then advance the advection-diffusion part of the equation $\Delta t$ in time followed by a second advancement of the reaction terms $\Delta t / 2$ in time.

The reaction part of the enthalpy and isotope equations are of the form

$$
\frac{\partial X_{k}}{\partial t}=\dot{\omega}_{k}
$$

and

$$
c_{p} \frac{\partial T}{\partial t}=-\sum_{k}\left(q_{k}+\xi_{k}\right) \dot{\omega}_{k}
$$

For the reaction phase, $c_{p}$ changes with temperature and composition; however, because of the computational expense associated with computing $c_{p}$ we have frozen its value for the integration of the ODE system. Numerical tests demonstrated that this simplification did not affect the computed deflagrations. As a result of this approximation, we do not use the updated temperature from the reaction step to update the enthalpy. Instead, we explicitly compute the change in enthalpy resulting from the change in isotope abundances and use this updated enthalpy to derive the correct temperature at the end of the reaction step.

In our implementation, we integrate the chemistry component using timeimplicit backward difference methods, as implemented in VODE [24], a generalpurpose stiff ODE integration software package. VODE utilizes adaptivity in order of accuracy and subcycled time-step selection so that an absolute error 
tolerance of $10^{-16}$ in mass fractions is maintained throughout. Typically, the resulting scheme is between third and fifth order convergent in time.

After completing the first reaction step, we update the advection-diffusion component of the system. One numerical issue that must be addressed at this point is the nonlinearity of the enthalpy diffusion. The advection-diffusion part of the enthalpy equation may be written explicitly in terms of enthalpy diffusion

$$
\frac{\partial \rho h}{\partial t}+\nabla \cdot U \rho h=\nabla \cdot \frac{\kappa}{c_{p}} \nabla h-\nabla \cdot\left(\sum_{k} \xi_{k} \frac{\kappa}{c_{p}} \nabla X_{k}\right)
$$

We advance this equation using a linear Crank-Nicolson algorithm, but the coefficients $\kappa$ and $c_{p}$ vary with the solution over time and space. These variations may be incorporated into the linear scheme simply by using a predictorcorrector iteration (detailed below), where the coefficients at the new-time are re-evaluated between iterations. With a good initial guess for the new-time $\kappa$ and $c_{p}$, a single corrector iteration is sufficient to guarantee stability and second-order accuracy in time.

We begin the advection-diffusion step with the cell-centered data (denoted with a superscript $n$ ) obtained from the initial chemistry advance. A secondorder Godunov procedure is used to extrapolate the temperature and abundances at $t^{n}$ to cell edges at $t^{n+1 / 2}=t+\Delta t / 2$. The fluid density at the edges is computed using the relation, $\rho=\sum_{k} \rho X_{k}$, and the enthalpy, $h$, is computed from $\rho, T$, and $X_{k}$. An explicit update for the new-time abundances at cellcenters, $\left(\rho X_{k}\right)^{n+1}$ may be formed using the extrapolated edge states, and the projected advection velocity, $U^{\mathrm{ADV}}$,

$$
\left(\rho X_{k}\right)^{n+1}=\left(\rho X_{k}\right)^{n}-\Delta t\left(\nabla \cdot U^{\mathrm{ADV}} \rho X_{k}\right)^{n+1 / 2}
$$

A corresponding cell-centered value of density at $t^{n+1}$ is then available using the expression

$\rho^{n+1}=\sum_{k}\left(\rho X_{k}\right)^{n+1}$.

Next, we predict a preliminary $t^{n+1}$ value of temperature, $\tilde{T}$, to be used in the initial estimates of the new-time transport coefficients. We employ a CrankNicolson discretization of the temperature equation with $t^{n}$ values of $\kappa$ and $c_{p}$.

$$
\rho^{n+1 / 2} c_{p}^{n}\left(\frac{\tilde{T}-T^{n}}{\Delta t}+\left(U^{\mathrm{ADV}} \cdot \nabla T\right)^{n+1 / 2}\right)=\frac{1}{2}\left(\nabla \kappa^{n} \nabla T^{n}+\nabla \kappa^{n} \nabla \tilde{T}\right)
$$


where $\rho^{n+1 / 2}=1 / 2\left(\rho^{n+1}+\rho^{n}\right)$. The new-time abundances and this preliminary temperature $\tilde{T}$ are then used to to evaluate provisional fluid properties $\left(\kappa, c_{p}, \xi_{k}\right)^{n+1, *}$. A predicted value of enthalpy $h^{n+1, *}$ is then computed using

$$
\begin{aligned}
\frac{\rho^{n+1} h^{n+1, *}-\rho^{n} h^{n}}{\Delta t}= & \left(\nabla \cdot U^{\mathrm{ADV}} \rho h\right)^{n+1 / 2} \\
& +\nabla \cdot \frac{1}{2}\left[\frac{\kappa^{n+1, *}}{c_{p}^{n+1, *}} \nabla h^{n+1, *}+\frac{\kappa^{n}}{c_{p}^{n}} \nabla h^{n}\right] \\
& -\nabla \cdot \frac{1}{2} \sum_{k}\left[\left(\xi_{k}^{n+1, *} \frac{\kappa^{n+1, *}}{c_{p}^{n+1, *}}\right) \nabla X_{k}^{n+1}+\left(\xi_{k}^{n} \frac{\kappa^{n}}{c_{p}^{n}}\right) \nabla X_{k}^{n}\right] .
\end{aligned}
$$

We complete the predictor component of our advance algorithm by extracting an updated provisional temperature, $T^{n+1, *}$, using Newton's method from $h^{n+1, *}$ and the $X_{k}^{n+1}$ values computed earlier.

The corrector step begins with a re-evaluation of $\kappa$ and $c_{p}$ using $T^{n+1, *}$ and $X_{k}^{n+1}$. The final enthalpy $h^{n+1}$ is obtained by solving

$$
\begin{aligned}
\frac{\rho^{n+1} h^{n+1}-\rho^{n} h^{n}}{\Delta t}= & \left(\nabla \cdot U^{\mathrm{ADV}} \rho h\right)^{n+1 / 2} \\
& +\nabla \cdot \frac{1}{2}\left[\frac{\kappa^{n+1}}{c_{p}^{n+1}} \nabla h^{n+1}+\frac{\kappa^{n}}{c_{p}^{n}} \nabla h^{n}\right] \\
& -\nabla \cdot \frac{1}{2} \sum_{k}\left[\left(\xi_{k}^{n+1} \frac{\kappa^{n+1}}{c_{p}^{n+1}}\right) \nabla X_{k}^{n+1}+\left(\xi_{k}^{n} \frac{\kappa^{n}}{c_{p}^{n}}\right) \nabla X_{k}^{n}\right]
\end{aligned}
$$

The temperature, $T^{n+1}$, is computed by once again inverting the equation of state for enthalpy, with $h^{n+1}$ and $X_{k}^{n+1}$. The integration of the enthalpy and abundance equations is completed by again advancing the reaction part of the system $\Delta t / 2$ in time. This provides a complete update of the $\rho, h, T$, and $X_{k}$ 's at the new time and allows us to evaluate the constraint on the constraint on the velocity field, $S^{n+1}$ at the new time.

The final step of basic integration step is to advance the velocity to the new time level. For this step we first obtain a provisional cell-centered velocity at $t^{n+1}$ using a time-lagged pressure gradient,

$$
\rho^{n+1 / 2} \frac{U^{n+1, *}-U^{n}}{\Delta t}+\rho^{n+1 / 2}\left[\left(U^{\mathrm{ADV}} \cdot \nabla\right) U\right]^{n+1 / 2}=-\nabla \pi^{n-1 / 2}+\rho^{n+1 / 2} \vec{g} .
$$

At this point $U^{n+1, *}$ does not satisfy the constraint. We apply an approximate projection to simultaneously update the pressure and to project $U^{n+1, *}$ onto 
the constraint surface. In particular, we solve

$$
L^{\rho} \phi=D\left(U^{n+1, *}+\frac{\Delta t}{\rho^{n+1 / 2}} G \pi^{n-1 / 2}\right)-S^{n+1}
$$

for nodal values of $\phi$, where $L^{\rho}$ is the standard bilinear finite element approximation to $\nabla \cdot \frac{1}{\rho} \nabla$ with $\rho$ evaluated at $t^{n+1 / 2}$. In this step, $D$ is a discrete secondorder operator that approximates the divergence at nodes from cell-centered data, and $G=-D^{T}$ approximates a cell-centered gradient from nodal data. In the formulation, $\phi$ satisfies Neumann boundary conditions at solid walls and inflow boundaries. At outflow boundaries, Dirichlet conditions are generated to suppress any tangential accelerations on the fluid leaving the domain. See Almgren et al. [25] for a more detailed discussion of projection issues. Nodal values for $S^{n+1}$ for the solution of (12) are computed using a volumeweighted average of cell-centered values. Finally, we determine the new-time cell-centered velocity field from

$$
U^{n+1}=U^{n+1, *}-\frac{\Delta t}{\rho^{n+1 / 2}}\left(G \phi-G \pi^{n-1 / 2}\right)
$$

and the new time-centered pressure from

$$
\pi^{n+1 / 2}=\phi .
$$

This completes the description of the time-advancement algorithm.

Before discussing the incorporation of this methodology in an adaptive mesh refinement algorithm, we note some of the properties of the algorithm. First, we emphasize that the temperature equation is used only in an auxiliary capacity in the algorithm. The energy is evolved using the numerically conservative discretized enthalpy equation, (10) and (11). As noted earlier, although the scheme rigorously satisfies conservation of mass and enthalpy, the evolution does not strictly maintain the equation of state at ambient pressure. Since the low Mach number asymptotics used to derive the governing equation show that the thermodynamic pressure only satisfies (1) to $O\left(M^{2}\right)$, relaxing the imposition of (1) is a reasonable way of dealing with the overdetermined system. However, to ensure that the accumulated deviation from the equation of state remains small over long-time integrations, we augment the constraint equation (5) to accommodate variations in thermodynamic pressure, and approximate those terms in such a way as to gently damp the deviation to zero. The appropriate material derivative of pressure, suitably scaled for addition to the right hand side of equation (5),

$$
\frac{f}{\gamma \rho \frac{\partial p}{\partial \rho}}\left(\frac{\partial p}{\partial t}+U \cdot \nabla p\right)
$$


is included during the intermediate velocity projection required to evaluate convective derivatives. In this expression $\gamma=c_{p} / c_{v}$ is the ratio of the two thermodynamic specific heats, and $f$ is a constant relaxation factor. We approximate $\partial p / \partial t$ by $\left(p_{\text {amb }}-p_{0}\right) / \Delta t$, where $p_{0}$ is defined discretely from equation (1), $p_{\text {amb }}$ is the specified ambient pressure, and $U \cdot \nabla p$ is approximated with upwind differences using $p_{0}$. Thus, we are effectively adding a first-order approximation to the material derivative of $p_{0}-p_{\text {amb }}$ along streamlines. This forcing term prevents the solution from deviating an appreciable amount from the equation of state while maintaining the second-order accuracy of the overall scheme.

\subsection{Adaptive mesh refinement}

In this section we present an overview of the adaptive projection algorithm. This framework, used in Day and Bell [14], was initially developed by Almgren et al. [26], and extended to low Mach number combustion by Pember et al. [27]. The discussion provides only an overview of the methodology. We refer the reader to the above papers for more details of the basic algorithm.

Our implementation of adaptive mesh refinement (AMR) is based on a sequence of nested grids with successively finer spacing in both time and space. In this approach, fine grids are formed by evenly dividing coarse cells by a refinement ratio, $r$, in each direction. Increasingly finer grids are recursively embedded in coarse grids until features of the solution are adequately resolved. An error estimation procedure based on user-specified criteria evaluates where additional refinement is needed and grid generation procedures dynamically create or remove rectangular fine grid patches as resolution requirements change.

The adaptive integration algorithm advances grids at different levels using time steps appropriate to that level, based on CFL considerations. The multi-level procedure can most easily be thought of as a recursive algorithm in which, to advance level $\ell, 0 \leq \ell \leq \ell_{\max }$, the following steps are taken:

- Advance level $\ell$ in time one time step, $\Delta t^{\ell}$, as if it is the only level. If $\ell>0$, obtain boundary data using time-interpolated data from the grids at $\ell-1$, as well as physical boundary conditions, where appropriate.

- If $\ell<\ell_{\max }$

- Advance level $(\ell+1)$ for $r$ time steps, $\Delta t^{\ell+1}=\frac{1}{r} \Delta t^{\ell}$, using level- $\ell$ data and the physical boundary conditions.

- Synchronize the data between levels $\ell$ and $\ell+1$, and interpolate corrections to finer levels $\left[\ell+2, \ldots, \ell_{\max }\right]$.

The adaptive algorithm, as outlined above, performs operations to advance 
the grids at each level independent of other levels in the hierarchy (except for boundary conditions) and then computes a correction to synchronize the levels. Loosely speaking, the objective in this synchronization step is to compute the modifications to the coarse grid that reflect the change in the coarse grid solution due to the presence of the fine grid. More specifically, when solving on a fine grid, we supply Dirichlet boundary conditions from the coarse grid. This leads to a mismatch in the associated fluxes at the coarse-fine interface that is corrected by the synchronization.

For the adaptive projection methodology presented here there are three basic steps in the synchronization. First, the values obtained for $U, \rho X_{k}$ and $\rho h$ are averaged from the fine grid onto the underlying coarse grid. We view the resulting data as defining a preliminary composite grid solution that is consistent between levels. We will denote this preliminary solution with a $p$ superscript in the remainder of the section. To complete the synchronization we need to correct inconsistencies arising from the use of Dirichlet boundary conditions at coarse-fine boundaries. In particular, we compute increments to $\rho X_{k}$ and $\rho h$ that correct the flux mismatches at coarse-fine interfaces. Finally, we correct the velocity field to satisfy a divergence constraint over the composite grid system.

There are two components that contribute to flux mismatch. First, $U^{\mathrm{ADV}}$, the edge-based advection velocity satisfies the constraint on the coarse level and the fine level separately. However, since we only satisfy the Dirichlet matching condition for $\phi^{\mathrm{MAC}}$ in (6), the value of $U^{\mathrm{ADV}}$ computed on the coarse level does not match the average value on the fine grid. We define the mismatch in advection velocities by

$$
\delta U^{\mathrm{ADV}, \ell}=-U^{\mathrm{ADV}, \ell, n+1 / 2}+\frac{1}{r^{2}} \sum_{k=0}^{r-1} \sum_{\text {edges }} U^{\mathrm{ADV}, \ell+1, n+k+1 / 2}
$$

along the coarse-fine boundary. We then solve the elliptic equation

$$
D^{\mathrm{MAC}} \frac{1}{\rho} G^{\mathrm{MAC}} \delta e^{\ell}=D^{\mathrm{MAC}} \delta U^{\mathrm{ADV}, \ell}
$$

and compute

$$
U^{\mathrm{ADV}, \ell, \mathrm{corr}}=-\frac{1}{\rho} G^{\mathrm{MAC}} \delta e^{\ell}
$$

which is the correction needed for $U^{\mathrm{ADV}}$ to satisfy the constraint and matching conditions on the composite $(\ell, \ell+1)$ grid hierarchy. This correction field is used to compute a modification to the advective fluxes for species and 
enthalpy that reflects an advection velocity field that satisfies the constraint on the composite grid.

The second part of the mismatch arises because the advective and diffusive fluxes on the coarse grid were computed without explicitly accounting for the fine grid, while on the fine grid the fluxes were computed using coarse-grid Dirichlet boundary data. We define the flux discrepancies

$$
\delta F_{\rho h}=\Delta t^{\ell}\left(-F_{\rho h}^{\ell, n+1 / 2}+\frac{1}{r^{2}} \sum_{k=0}^{r-1} \sum_{\text {edges }} F_{\rho h}^{\ell+1, n+k+1 / 2}\right)
$$

and

$$
\delta F_{\rho X_{k}}=\Delta t^{\ell}\left(-F_{\rho X_{k}}^{\ell, n+1 / 2}+\frac{1}{r^{2}} \sum_{k=0 \text { edges }}^{r-1} \sum_{\rho X_{k}}^{\ell+1, n+k+1 / 2}\right)
$$

where $F$ is the total (advective+diffusive) flux through a given interface prior to these synchronization operations. Since mass is conserved, corrections to density, $\delta \rho^{\text {sync }}$, on the coarse grid associated with mismatched advection fluxes may be computed explicitly

$$
\delta \rho X_{k}^{\mathrm{sync}}=-D^{\mathrm{MAC}}\left(U^{\mathrm{ADV}, \mathrm{corr}} \rho X_{k}\right)^{n+1 / 2}+\delta F_{\rho X_{k}} .
$$

and $\delta \rho^{\mathrm{sync}}=\sum_{k} \delta \rho X_{k}^{\mathrm{sync}}$.

The synchronization correction for $h$ is more complex because of the implicit discretization of diffusion. In particular, computing $\delta h^{\text {sync }}$ requires solution of a linear system, since the flux mismatch contains implicit diffusion fluxes from the Crank-Nicolson discretization. To set up the synchronization, we first note that

$$
\delta(\rho h)^{\mathrm{sync}}=h^{n+1, p} \delta \rho^{\mathrm{sync}}+\rho^{n+1} \delta h^{\mathrm{sync}} .
$$

Then, we have

$$
\begin{aligned}
\left(\rho^{n+1}-\frac{\Delta t}{2} \nabla \frac{\kappa^{n+1}}{c_{p}^{n+1}} \nabla\right) \delta h^{\mathrm{sync}}= & -D^{\mathrm{MAC}}\left(U^{\mathrm{ADV}, \mathrm{corr}} \rho h\right)^{n+1 / 2}+\delta F_{\rho h} \\
& +\nabla \cdot \sum_{k} \xi_{k}\left(T^{n+1, p}\right)\left(\frac{\kappa^{n+1}}{c_{p}^{n+1}} \nabla \delta X_{k}^{\mathrm{sync}}\right) .
\end{aligned}
$$

The corrections $\delta \rho^{\mathrm{sync}}, \delta \rho X_{k}^{\mathrm{sync}}$, and $\delta \rho h^{\mathrm{sync}}$ are added to the coarse field at 
level- $\ell$, and interpolated to all finer levels. Finally, a new temperature field is computed using Newton's method on all affected levels.

A similar process is also used to generate a correction to the velocity field. However, the velocity flux correction must be projected to obtain the component satisfying the constraint that updates $U$ and the component that updates $\pi$. At this point there are two additional corrections needed for the composite velocity field:

- A correction arising because the projection at level $\ell+1$ used Dirichlet data from level $\ell$, leading to a mismatch in normal derivative at coarse-fine boundaries

- The temperature and species adjustment in the first part of the synchronization leads to an increment in the computed $S$ field.

Since the projection is linear, both of these corrections as well as the projection of the velocity flux correction can be combined into a single, multi-level nodebased synchronization solve performed at the end of a coarse-grid time step.

We note that with the synchronization procedure outlined above, the adaptive algorithm preserves the second-order accuracy and the conservation properties of the single-grid algorithm. The methodology has been implemented for distributed memory parallel processors using the BoxLib class libraries described by Rendleman et al. [28]. In this approach, grid patches are distributed to processors using a heuristic knapsack algorithm to balance the computational work developed by Crutchfield [29] (see also, Rendleman et al. [28]).

\section{Results}

In this section we present two sets of computational results. The first set of results presents comparisons of the low Mach number model with a comparable compressible code for one-dimensional flames at various densities. These examples serve to validate the low Mach number algorithm and quantify the errors associated with the low Mach number approximation. The second set of results describes the application of the methodology to simulation of the Landau-Darrieus instability in two dimensions.

The numerical simulations were performed using the equation of state described by Timmes and Swesty [30] which computes the internal energy, pressure and thermodynamic derivatives (including the specific heats at constant volume and pressure) of these quantities as functions of temperature, density and the nuclear-species mass fractions. The values of the thermal conductivity, $\kappa$, are calculated using the procedure described by Timmes [31]. 


\subsection{Validation}

The validation studies were performed by comparing one-dimensional laminar solutions tabulated in Dursi et al. [32] to ${ }^{12} \mathrm{C} /{ }^{24} \mathrm{Mg}$ nuclear flames for several physical conditions. Each simulation was constructed in the same way. A twodimensional domain, periodic in one dimension, and with an inflow boundary condition on one face and an outflow condition on the opposite face is constructed. For each case we initialize the domain with an interface separating ${ }^{12} \mathrm{C}$ fuel and ${ }^{24} \mathrm{Mg}$ ash. We specify density and temperature for the ${ }^{12} \mathrm{C}$ and temperature for the ${ }^{24} \mathrm{Mg}$. For this specification, the temperature of the fuel must be below the initiation temperature for the reaction whereas the initial ash temperature must be high enough to ignite the flame. The ash density is computed from the equation of state so that pressure is constant in the domain, consistent with the low Mach number hypothesis. We specify inflow of the cold fuel at a fixed speed on the ${ }^{12} \mathrm{C}$ side of the interface and specify outflow on the ${ }^{24} \mathrm{Mg}$ side. The region of contact between the fuel and the ash is smoothed over a distance that is a small fraction of the size of the computational domain. The grid spacing is specified so that there are approximately 5 computational zones, at the coarsest level of refinement, in the flame.

The simulation proceeds with a single level of refinement until the initiation of the nuclear flame which is seen as a deviation of the temperature of the outflowing ash and a sharp increase in the energy generation rate. At that point, additional levels of refinement are added to the simulation until the speed of the nuclear flame and the flame shape converges. The simulation is then restarted using this computed constant speed to obtain a steady laminar solution.

To model the ${ }^{12} \mathrm{C} /{ }^{24} \mathrm{Mg}$ reaction we used a single-step mechanism derived from Caughlan and Fowler [33]. This reaction has the form:

$$
\begin{aligned}
\dot{X}_{{ }_{12} \mathrm{C}}(t) & =-\frac{1}{12} R(T) \rho X_{12}^{2}(t) \\
\dot{T}(t) & =-Q \dot{X}_{{ }_{12} \mathrm{C}}(T) / c_{p} .
\end{aligned}
$$

where the rate of reaction, $R(T)$, is

$$
R(T)=4.27 \cdot 10^{26} \frac{T_{9, a}^{5 / 6}}{T_{9}^{3 / 2}} \exp \left\{\frac{-84.165}{T_{9, a}^{1 / 3}}-2.12 \cdot 10^{-3} T_{9}^{3}\right\},
$$

$T_{9}=T / 10^{9} \mathrm{~K}, T_{9, a}=T_{9} /\left(1+0.0396 T_{9}\right), \rho$ is density, and $Q$ is a constant. For the ${ }^{12} \mathrm{C} /{ }^{24} \mathrm{Mg}$ reaction, the value of the specific energy release, $Q$, is taken to be $5.57 \cdot 10^{17} \mathrm{erg} / \mathrm{g}$. As noted above, the specific heat can be held constant 
with no apparent loss of accuracy. We neglect the effects of nuclear screening on this rate, as they are quite small for the conditions we consider.

Two ${ }^{12} \mathrm{C} /{ }^{24} \mathrm{Mg}$ simulations with initial ${ }^{12} \mathrm{C}$ density values of $\rho=2.5$ and $5 \cdot 10^{7} \mathrm{~g} / \mathrm{cm}^{3}$, both with a fuel temperature of $10^{7} \mathrm{~K}$ were run. Measured laminar flame speeds differed by no more that .1\% from those listed in Dursi et al. [32]. Figure 1 shows the laminar flame solution for temperature, density and flow velocity for the case in which the initial ${ }^{12} \mathrm{C}$ density is $5 \cdot 10^{7} \mathrm{~g} / \mathrm{cm}^{3}$. The slight differences in the computed flame speed between FLASH and the low Mach number code result in slightly different flame locations after the flame has relaxed to a full steady state. We have corrected for this effect by spatially shifting the FLASH solutions for comparison to the low Mach number results. In Figure 1 we plot the computed laminar flame solution and the difference between low Mach number solution and the solution computed with FLASH The largest difference is in temperature which shows an error of approximately $1.25 \%$ in the transition region where temperature changes by almost three orders of magnitude. Differences between the solutions for density and flow velocity are less than $1 \%$.

Finally, we make some additional remarks concerning the relative efficiency of the low Mach number method compared to the compressible methods. The low Mach number method in this paper and the compressible method used in FLASH have similar strategies for resolving spatial structures in the fluid flow. Therefore, they tend to result in similar resolutions in their spatial discretizations. Where they differ is in the time-step requirements. The time steps in a compressible method are limited by the need to obey the CFL constraint using as a velocity the speed of sound in the fluid; the low Mach number method has a similar CFL constraint, however it uses the fluid advection velocity. For the case considered in this section, the speed of sound is approximately $5 \cdot 10^{8} \mathrm{~cm} / \mathrm{s}$, while a typical advective velocity is the laminar flame speed, which is approximately $6.82 \cdot 10^{5} \mathrm{~cm} / \mathrm{s}$. Thus, the low Mach number implementation requires roughly a factor of 1000 fewer time steps to model the same flow. For lower density flames, and flames that contain more than one species, this disparity can be even greater. For example, a flame consisting of $75 \%{ }^{12} \mathrm{C}$ and $25 \%{ }^{16} \mathrm{O}$ at a density of $2.5 \cdot 10^{7} \mathrm{~g} / \mathrm{cm}^{3}$ has a ratio of sound speed to laminar speed of nearly 5000 .

\subsection{Landau-Darrieus simulations}

In this section we describe simulations designed to illustrate the LandauDarrieus (LD) instability in a nuclear flame using direct numerical simulation. A perturbed initial planer inflow of ${ }^{12} \mathrm{C}$ fuel impinges on a hot, lower density ${ }^{24} \mathrm{Mg}$ ash. As above, the fuel burns in a single-step mechanism to form the ash. 


\section{Temperature}
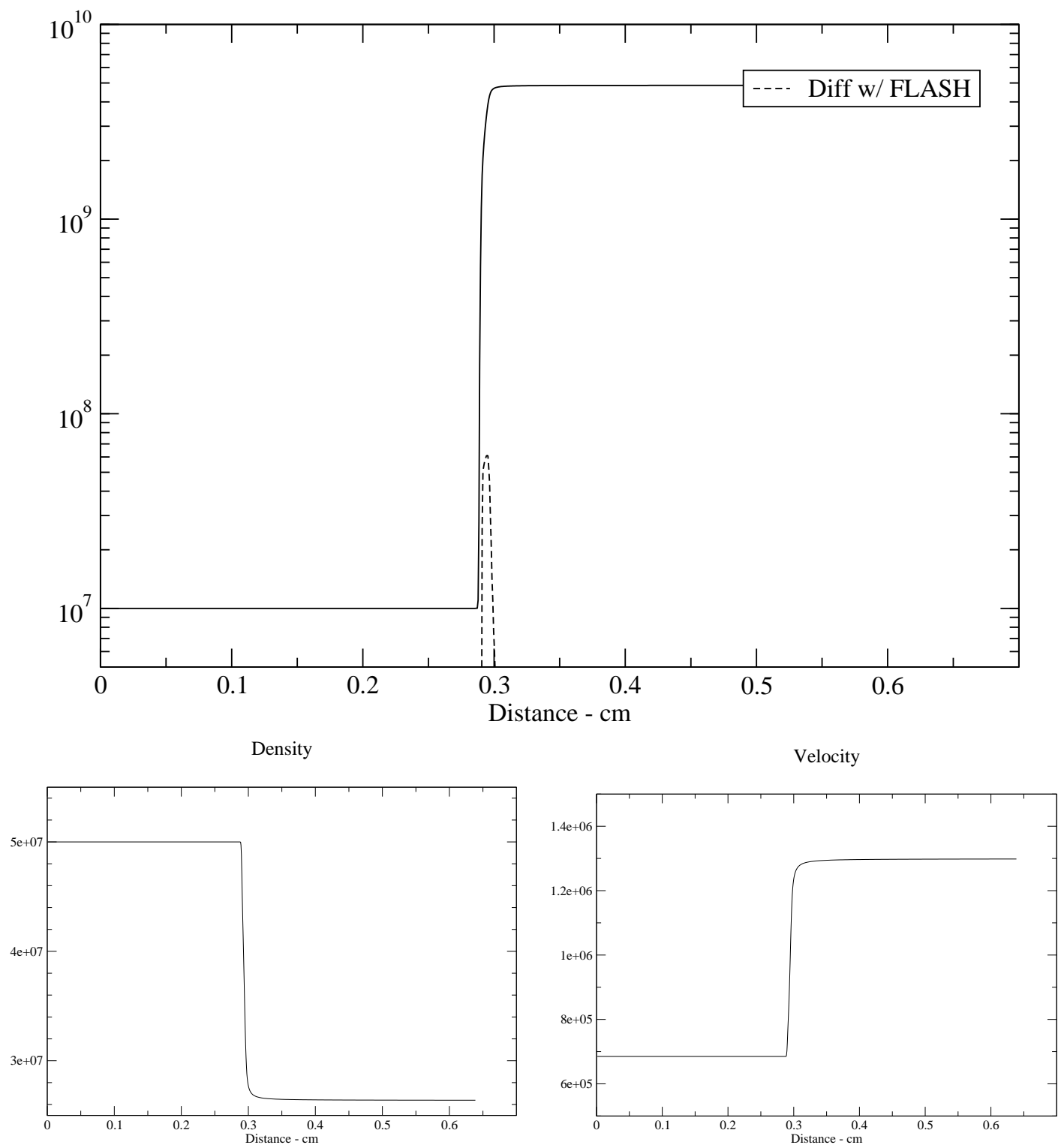

Fig. 1. Laminar flame solution for $\rho=5 \cdot 10^{7} \mathrm{~g} / \mathrm{cm}^{3}, T=10^{7} \mathrm{~K}$. Shown are the solutions for density, speed, and temperature. The difference with the FLASH results from Dursi et al. [32] is indicated on the temperature result

The initial perturbation is formed by shifting the laminar flame solution for the corresponding density, temperature, and mass fractions such that a fixed number of wavelengths of random phase and amplitude are contained in the domain.

Before illustrating the application of the method discussed above to the dynamics of multi-mode Landau-Darrieus instabilites, we first present a twodimensional convergence study for a single mode in a smaller domain to as- 


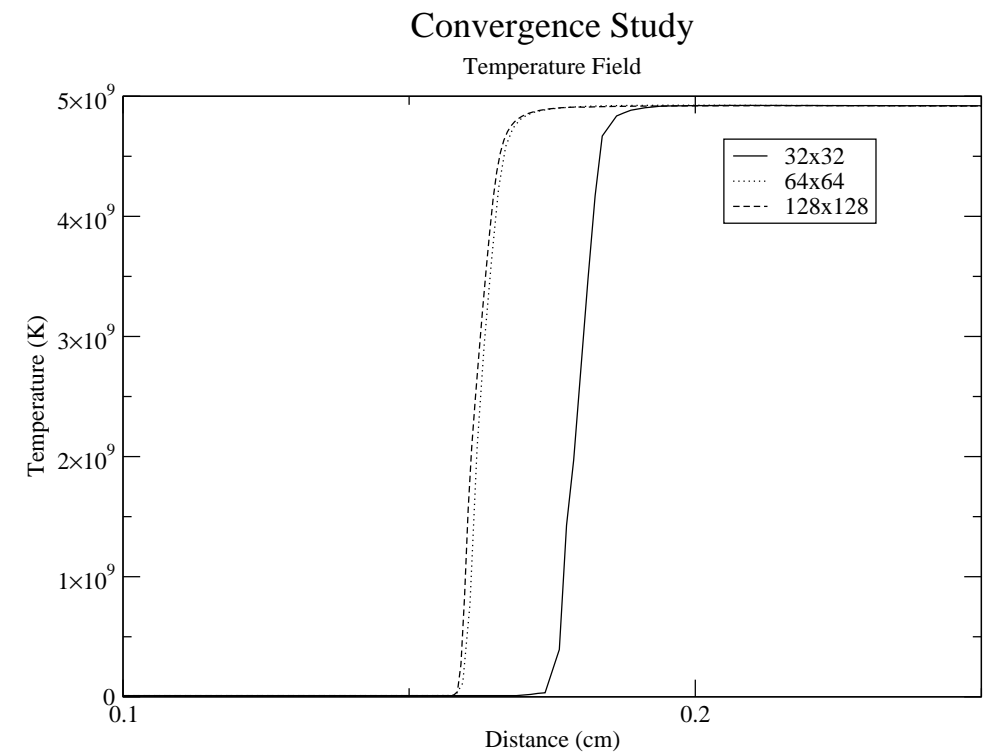

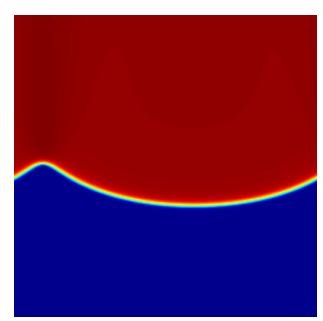

$64 \times 64$

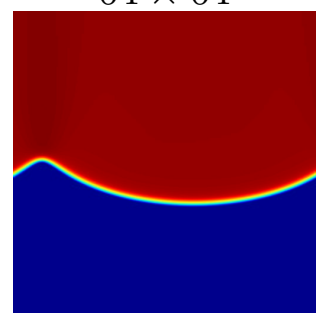

$128 \times 128$

Fig. 2. Two-dimensional convergence study. Left: Curves represent temperature versus distance along a vertical slice through the cusp for base resolutions of $32 \times 32$, $64 \times 64$, and $128 \times 128$, with 2 levels of refinement $.420 \mu$ s. Right: image of temperature field at medium and finest resolutions.

sess the resolution requirements needed for accurate multidimensional simulations. For this study we initialize with a single frequency in a small domain of $.32 \times .32 \mathrm{~cm}^{2}$ at 3 resolutions corresponding to base grids of $32 \times 32,64 \times 64$, and $128 \times 128$ using 2 levels of refinement. Figure 2 demonstrates convergence of the method and shows that acceptable accuracy is obtained on the $64 \times 64$ base grid, which corresponds to approximately 5 points per flame thickness at the grid spacing of the finest level. The flame thickness is defined to be $\left(T_{\max }-T_{\min }\right) / \max (\nabla T)$. (Another common definition for the flame thickness is the width of the zone $10 \%-90 \%$ of the peak temperature, Timmes and Woosley [19], which results in flame thickness about twice as large.) For the computations presented below we have used a resolution comparable to the $128 \times 128$ case.

Figure 3 illustrates the LD instability by showing the time history of velocity field. In this calculation the random perturbation of the initial planar laminar solution contained 30 frequencies of amplitude approximately 50 times the laminar flame thickness. The domain is $2.56 \times 1.28 \mathrm{~cm}^{2}$ with $1024 \times 512$ zones at the coarsest level of refinement. Cells with steep temperature gradients were refined up to two levels giving an effective computational domain of $4096 \times 2048$ zones. The density of the ${ }^{12} \mathrm{C}$ fuel is $5 \cdot 10^{7} \mathrm{~g} / \mathrm{cm}^{3}$ and the inflow temperature is $10^{7} \mathrm{~K} ;{ }^{12} \mathrm{C}$ fuel is being passed in from the bottom into the ash that is at the top of the figure (i.e. the center of the star is above the top of the figure). In this figure, the letters A and B mark two cusps that slowly coalesce to form a single LD cusp. This behavior was also seen in the $\mathrm{LD}$ calculation described next, and we conjecture that in periodic domains LD 

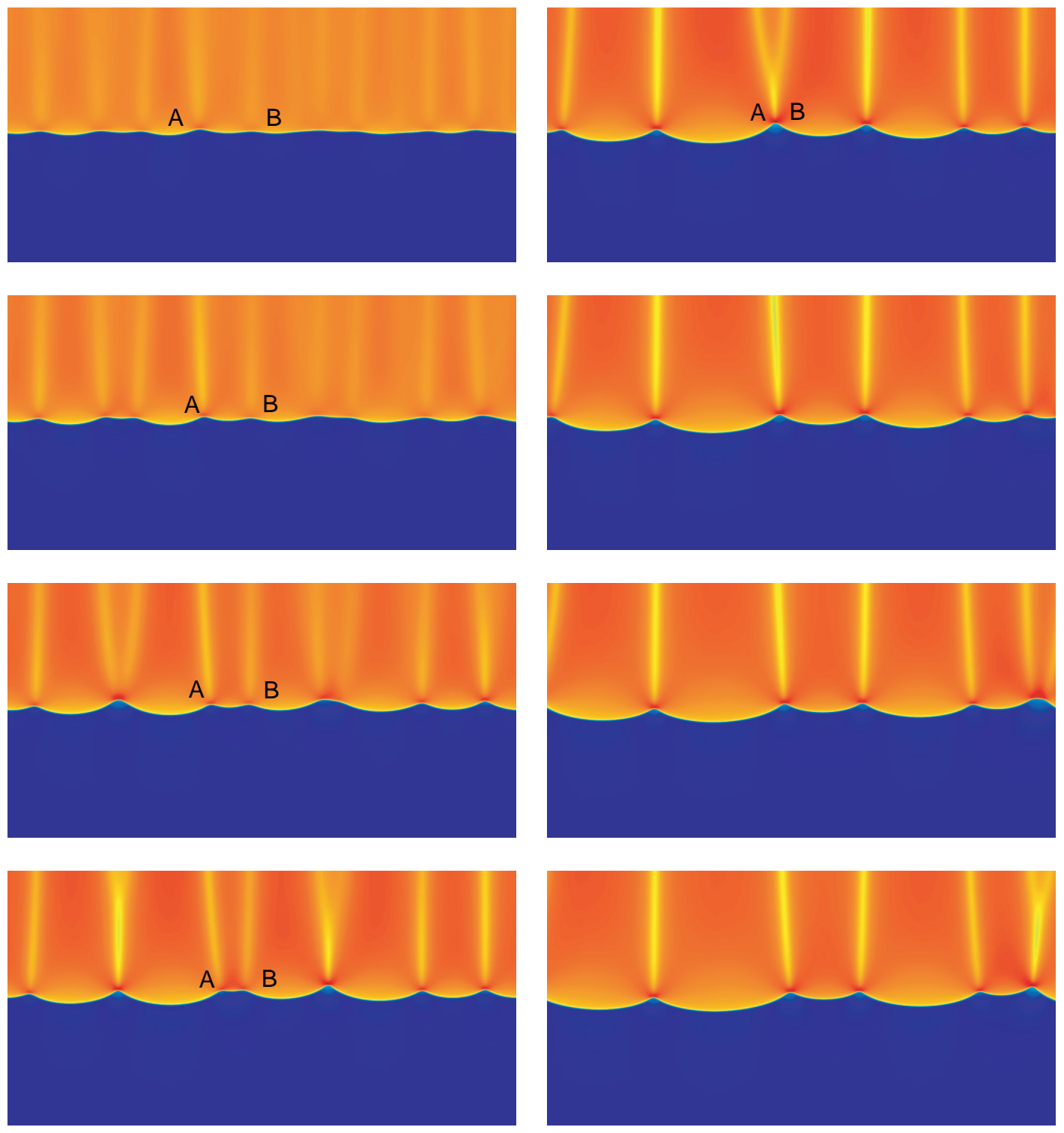

Fig. 3. Time history of LD simulation, showing coalescence of LD peaks (e.g., A and $\mathrm{B}) ; \rho=5 \cdot 10^{7} \mathrm{~g} / \mathrm{cm}^{3}$ and $T=10^{7} \mathrm{~K}$. Shown is vertical flow velocity. The 'searchlights' are regions of lower flow speed in the ash (see Figure 4.) Time increases down the left hand column from .78 $\mu$ s and continues down the right column to $3.4 \mu \mathrm{s}$.

cusps will always coalesce until only one cusp remains. Figure 4 explains the appearance of the 'searchlight' features in Figure 3. Although the flow speed in the ash is sharply peaked at the LD cusp, it rapidly decays to a speed that is lower than the post-flame speed in the valleys between the cusps. The flow speed in the fuel is depressed the valleys between the cusps relative to the flow speed in the fuel below the LD cusps.

Figure 5 shows a well developed LD cusp for a simulation performed on a 


\section{Flow Velocity $-\mathrm{cm} / \mathrm{s}$}

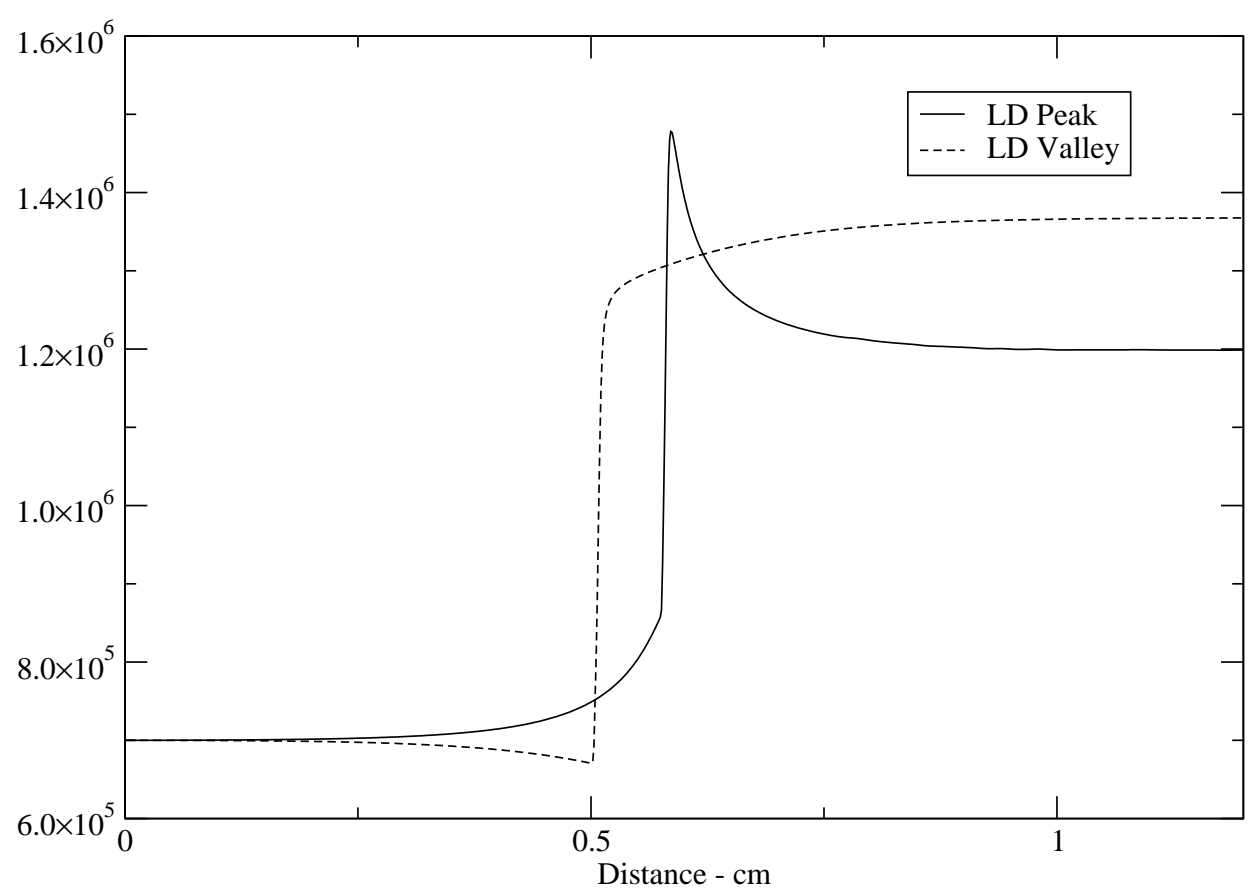

Fig. 4. Flow velocity at peak of the LD cusp and valleys between the LD cusps

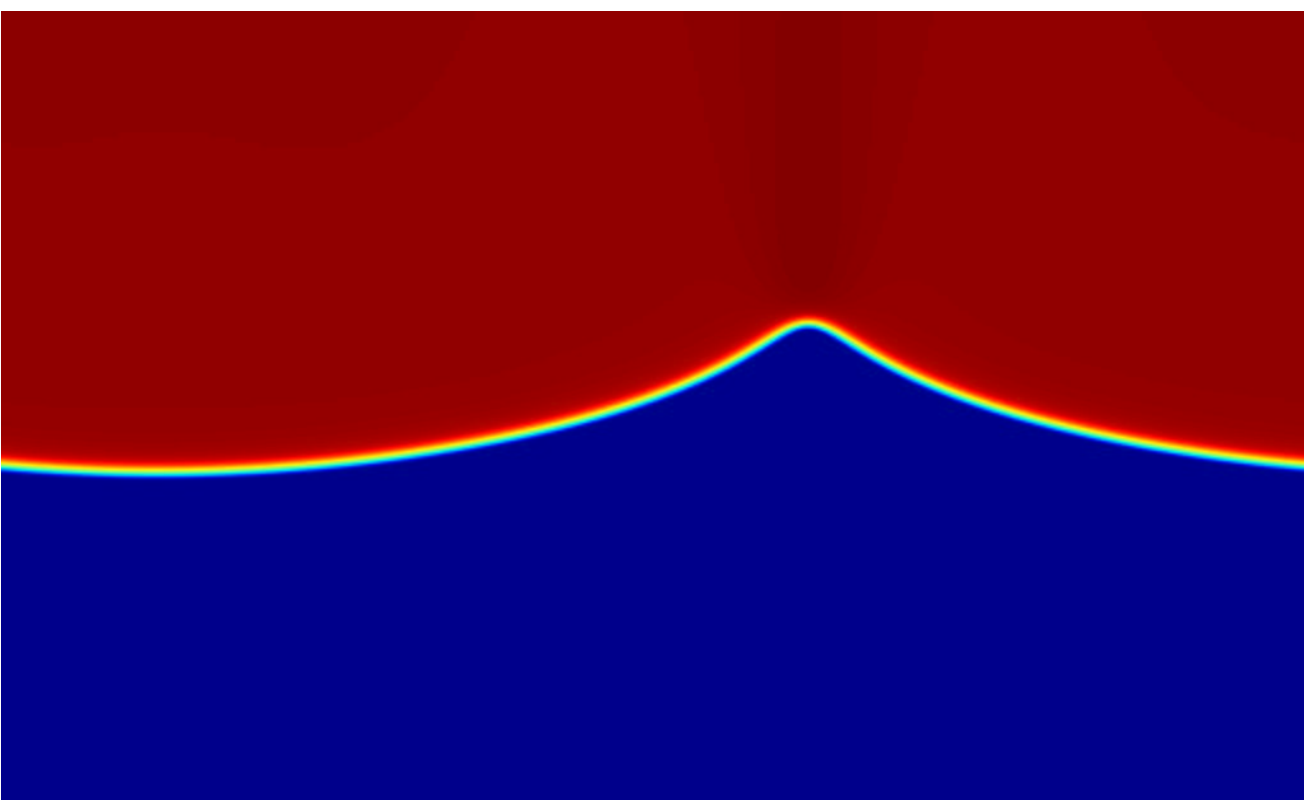

Fig. 5. Landau Darrieus Cusp: ${ }^{12} \mathrm{C}, \rho=5 \cdot 10^{7} \mathrm{~g} / \mathrm{cm}^{3}, T=10^{7} \mathrm{~K} ; t=10 \mu \mathrm{s}$.

smaller domain $\left(1.28 \times .64 \mathrm{~cm}^{2}\right.$ at base resolution of $\left.512 \times 256\right)$ using the same material parameters as in the previous example. In this case, only 5 frequencies were used to randomize the planar laminar solution. After about $2-3 \mu$ s the details of the initial perturbations have disappeared and the LD cusps have coalesced. We continued to track the solution up to $10 \mu$ s. Figure 6 shows the 


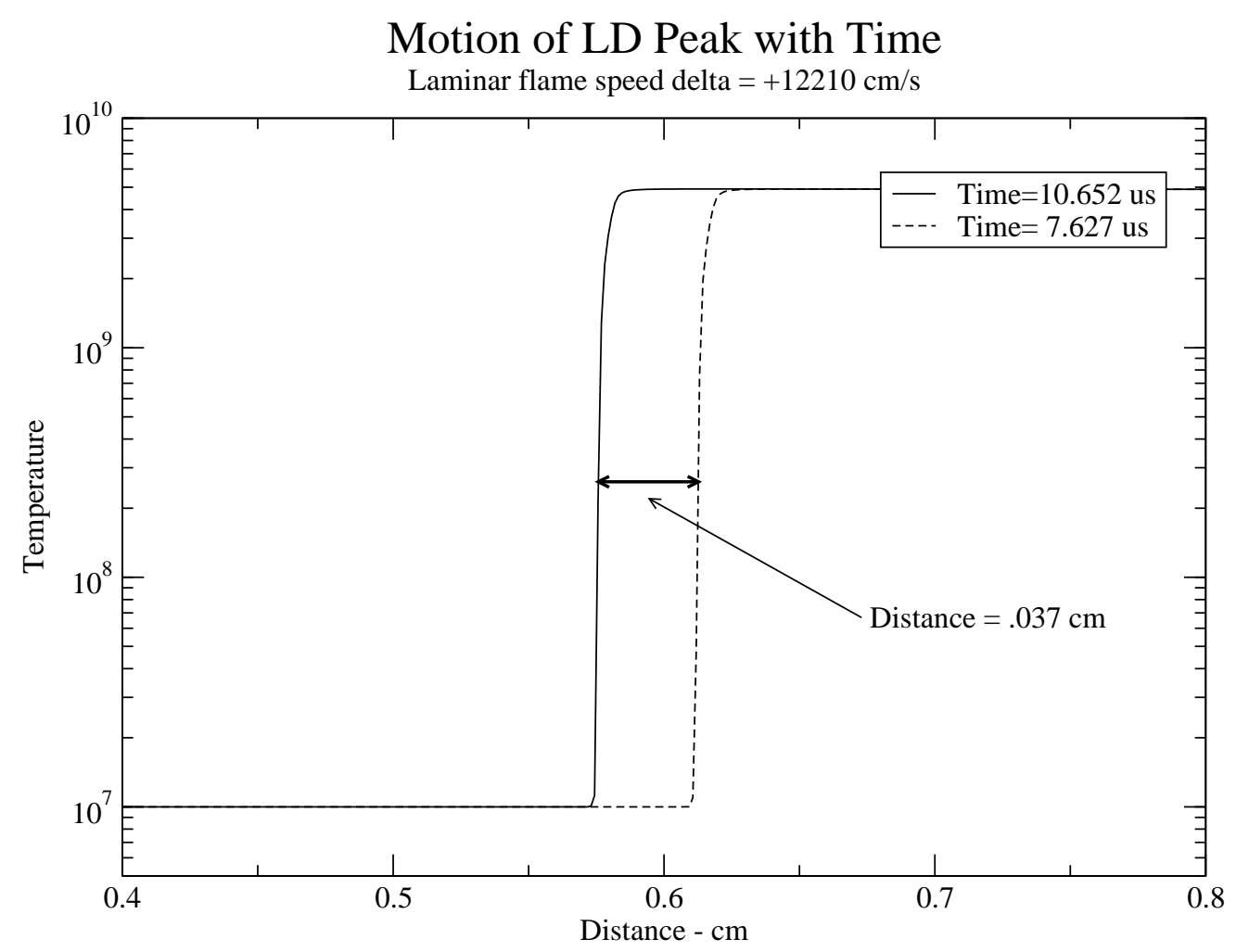

Fig. 6.

displacement in the stabilized LD cusp over a time range of approximately $3 \mu \mathrm{s}$, indicating a increase in the laminar speed of $12,210 \mathrm{~cm} / \mathrm{s}$, or about $1.8 \%$. Over the time period 3-10 $\mu$ s the amplitude of the cusp decreases by $.009 \mathrm{~cm}$, which is approximately $10 \%$ of the extent of the cusp at $t=3 \mu \mathrm{s}$. The long time behavior of an isolated cusp is under investigation.

We note that the behavior of the flame undergoing the Landau-Darrieus instability at this density is considerable smoother than that shown in Niemeyer and Hillebrandt [5] for the same density. The reason for this difference is that we initialize the problem in pressure equilibrium whereas Niemeyer and Hillebrandt do not. Consequently, in the Niemeyer and Hillebrandt study there is an initial transient phase in which the acoustic waves relax in the domain. (This relaxation occurs on a much faster time scale than the flame propagation.) As a result, their flame propagates into region where the velocity field contains fine-scale remnants of the relaxation whereas in our simulations the flame propagates into an undisturbed region.

Several mechanisms have been proposed [1] that could give rise to an acceleration of the laminar flame speed in a Type Ia supernova. One part of some of these mechanisms is that the LD instability through the wrinkling in the flame surface, while not giving rise to turbulent motion, could give rise to sufficient flame speed acceleration to account for observed isotopic abundances and energy release. These calculations seem to indicate the LD instability by 
itself is insufficient to give rise to significant acceleration of the flame front.

\section{Conclusions}

The low Mach number numerical methods for chemical combustion introduced by Day and Bell [14] has been successfully extended to account for non-ideal gas law equations of state. The method yields results that compare well with established compressible simulations (Dursi et al. [32]). The low Mach number method enables new astrophysical problems to be explored, such as fully resolved instabilities at low-moderate densities; such problems are not tractable with a fully compressible code.

The computer program implementing the algorithms presented in this paper will be used to conduct several sets of computational experiments aimed at increasing our understanding of the microphysics of nuclear flames. Though not detailed in the presentation, the program has already been extended to handle more than one nuclear reaction, more than two isotopes, and three dimensions. This code will be used to perform a comprehensive examination of the phenomenology of two dimensional instabilities of the flame front. Later, the effects of Landau-Darrieus and Rayleigh-Taylor instabilities and their interaction with turbulence will be examined in three dimensions.

\section{Acknowledgments}

We thank F. X. Timmes for making his equation of state and conductivity routines available online.

\section{References}

[1] W. Hillebrandt, J. C. Niemeyer, Type Ia supernova explosion models, Annu. Rev. Astron. Astrophys 38 (2000) 191-230.

[2] L. D. Landau, On the theory of slow combustion, Acta Physicochimica, USSR 19 (1944) 77-85.

[3] G. Darrieus, Propagation d'un front de flamme, in: La Technique Moderne, France, 1938.

[4] S. I. Blinnikov, P. V. Sasorov, Landau-Darrieus instability and the fractal dimension of flame fronts, Phys. Rev. E 53 (1996) 4827-4841. 
[5] J. C. Niemeyer, W. Hillebrandt, Microscopic instabilities of nuclear flames in type ia supernovae, Astrophysical Journal 452 (1995) 779-784.

[6] A. M. Khokhlov, Propagation of turbulent flames in supernovae, Astrophysical Journal 449 (1995) 695.

[7] J. C. Niemeyer, W. Hillebrandt, Microscopic and macroscopic modeling of thermonuclear burning fronts, in: P. Ruiz-Lapuente, R. Canal, R. Isern (Eds.), Thermonuclear Supernovae, Dordrecht:Kluwer, 1997.

[8] J. C. Niemeyer, W. K. Busche, G. R. Ruetsch, Small-scale interaction of turbulence with thermonuclear flames in type ia supernovae, Astrophysical Journal 524 (1999) 290.

[9] B. Fryxell, K. Olson, P. Ricker, F. X. Timmes, M. Zingale, D. Q. Lamb, P. MacNeice, R. Rosner, J. W. Truran, H. Tufo, FLASH: An Adaptive Mesh Hydrodynamics Code for Modeling Astrophysical Thermonuclear Flashes, Astrophysical Journal Supplement 131 (2000) 273-334.

[10] A. C. Calder, B. Fryxell, T. Plewa, R. Rosner, L. J. Dursi, V. G. Weirs, T. Dupont, H. F. Robey, J. O. Kane, B. A. Remington, R. P. Drake, G. Dimonte, M. Zingale, F. X. Timmes, K. Olson, P. Ricker, P. MacNeice, H. M. Tufo, On Validating an Astrophysical Simulation Code, Astrophysical Journal Supplement 143 (2002) 201-229.

[11] R. Rehm, H. Baum, The equations of motion for thermally driven buoyant flows, N.B.S.J.Res. 83 (1978) 297-308.

[12] A. Majda, J. A. Sethian, The derivation and numerical solution of the equations for zero Mach number combustion, Combust. Sci. Technol. 42 (1985) 185-205.

[13] O. Knio, H. N. Najm, P. S. Wyckoff, A semi-implicit numerical scheme for reacting flow. II. Stiff, operator split formulation, J. Comput. Phys. 154 (1999) $428-467$.

[14] M. S. Day, J. B. Bell, Numerical simulation of laminar reacting flows with complex chemistry, Combust. Theory Modelling 4 (4) (2000) 535-556.

[15] B. Bennett, M. D. Smooke, A comparison of the structures of lean and rich axisymmetric laminar bunsen flames: application of local rectangular refinement solution-adaptive gridding, Combust. Theory Modelling 3 (1999) 657-687.

[16] R. Becker, M. Braack, R. Rannacher, Numerical simulation of laminar flames at low mach number by adaptive finite elements, Combust. Theory Modelling 3 (1999) 503-534.

[17] J. B. Bell, N. J. Brown, M. S. Day, M. Frenklach, J. F. Grcar, S. R. Tonse, The dependence of chemistry on the inlet equivalence ratio in vortex-flame interactions, Proc. Combust. Inst. 28 (2000) 1933-1939.

[18] J. B. Bell, M. S. Day, J. F. Grcar, M. J. Lijewski, Numerical simulation of premixed turbulent methane combustion, Tech. Rep. LBNL-49331, Lawrence Berkeley National Labortory, submitted to Proceedings of the Combustion Institute (2002). 
[19] F. X. Timmes, S. E. Woosley, The conductive propagation of nuclear flames i. degenerate $\mathrm{C}+0$ and $\mathrm{O}+\mathrm{Ne}+\mathrm{Mg}$ white dwarfs, Astrophysical Journal 396 (1992) 649-667.

[20] R. Kippenhahn, A. Weigert, Stellar Structure and Evolution, Springer Verlag, 1992.

[21] P. McMurtry, W.-H. Jou, J. Riley, R. Metcalfe, Direct numerical simulations or a reacting mixing layer with chemical heat release, AIAA J. 24 (1986) 962.

[22] F. Nicoud, Conservative high-order finite-difference schemes for low-Mach number flows, J. Comput. Phys. 158 (2000) 71-97.

[23] F. H. Harlow, J. E. Welch, Numerical calculation of time-dependent viscous incompressible flow of fluids with free surfaces, Physics of Fluids 8 (1965) 21822189 .

[24] P. N. Brown, G. D. Byrne, A. C. Hindmarsh, VODE: A variable coefficient ode solver, SIAM J. Sci. Stat. Comput. 10 (1989) 1038-1051.

[25] A. S. Almgren, J. B. Bell, W. Y. Crutchfield, Approximate projection methods: Part I. Inviscid analysis, SIAM J. Sci. Comput. 22 (4) (2000) 1139-59.

[26] A. S. Almgren, J. B. Bell, P. Colella, L. H. Howell, M. Welcome, A conservative adaptive projection method for the variable density incompressible NavierStokes equations, J. Comput. Phys. 142 (1998) 1-46.

[27] R. B. Pember, L. H. Howell, J. B. Bell, P. Colella, W. Y. Crutchfield, W. A. Fiveland, J. P. Jessee, An adaptive projection method for unsteady, low-Mach number combustion, Comb. Sci. Technol. 140 (1998) 123-168.

[28] C. A. Rendleman, V. E. Beckner, M. Lijewski, W. Y. Crutchfield, J. B. Bell, Parallelization of structured, hierarchical adaptive mesh refinement algorithms, Computing and Visualization in Science 3 (3) (2000) 147-157.

[29] W. Y. Crutchfield, Load balancing irregular algorithms, Tech. Rep. UCRL-JC107679, Lawrence Livermore National Laboratory (Jul. 1991).

[30] F. X. Timmes, F. D. Swesty, The accuracy, consistency, and speed of an electronpositron equation of state based on table interpolation of the helmholtz free energy, Astrophysical Journal Supplement 126 (2000) 501-516.

[31] F. X. Timmes, The physical properties of laminar helium deflagrations, Astrophysical Journal 528 (2000) 913.

[32] L. J. Dursi, M. Zingale, A. C. Calder, B. Fryxell, F. X. Timmes, N. Vladimirova, R. Rosner, A. Caceres, D. Q. Lamb, K. Olson, P. M. Ricker, K. Riley, A. Siegel, J. W. Truran, The Response of Astrophysical Thermonuclear Flames to Curvature and Stretch, Astrophysical Journal 595 (2003) 955-979.

[33] G. R. Caughlan, W. A. Fowler, Thermonuclear reaction rates V, Atomic Data and Nuclear Data Tables 40 (2) (1988) 283-334, see also http://www.phy. ornl.gov/astrophysics/data/cf88/index.html. 